(Figure 2, $B$ and $C$ ). The patient's dyspnea has since resolved. For the past 18 months, the patient has had no evidence of disease with regular follow-ups with surveillance CT scans.

\section{DISCUSSION}

Recurrent metastatic disease after nephrectomy for RCC is relatively common, occurring in $20 \%$ to $50 \%$ of patients. The prognosis of metastatic RCC is poor, demonstrating a 6- to 12 -month median overall survival and a $10 \%$ to $20 \%$ 2-year overall survival. ${ }^{2}$ The lungs are the most common location for RCC metastases, accounting for the location of $45 \%$ to $75 \%$ of all metastatic disease. ${ }^{3}$ Pulmonary metastasectomy appears to yield favorable results in certain patients, although these individuals largely have a high performance status with generous pulmonary reserve. Moreover, the biology of the cancer is important, because patients with shorter disease-free intervals and those with large $(>3 \mathrm{~cm})$ metastases have poorer outcomes.

Pleural metastases of RCC are relatively rare. ${ }^{4,5}$ Autopsy data have demonstrated that $12 \%$ of all patients with metastatic RCC have pleural involvement, involvement that is invariably associated with pulmonary metastasis. ${ }^{4}$
While cases of pleural RCC metastasis are generally thought to occur from direct extension of lung parenchymal RCC metastases, some cases of pleural RCC, such as that described here, occur without lung metastases. In such cases, it has been suggested that pleural metastases may occur by way of the Batson venous plexus. ${ }^{5}$ Although pulmonary metastasectomy is considered by many to be reasonable for metastatic parenchymal RCC, a pleurectomy and decortication procedure for pleural RCC metastases has never been reported to the best of our knowledge. We present here a case study of a pleurectomy and decortication procedure for pleural metastatic RCC that provided excellent symptom relief and local control.

\section{References}

1. Sugarbaker DJ, Wolf AS. Surgery for malignant pleural mesothelioma. Expert Rev Respir Med. 2010;4:363-72.

2. Flanigan RC, Campbell SC, Clark JI, Picken MM. Metastatic renal cell carcinoma. Curr Treat Options Oncol. 2003;4:385-90.

3. Kim DY, Karam JA, Wood CG. Role of metastasectomy for metastatic renal cell carcinoma in the era of targeted therapy. World J Urol. 2014;32:631-42.

4. Saitoh H. Distant metastasis of renal adenocarcinoma. Cancer. 1981;48: 1487-91.

5. Ohnishi H, Abe M, Hamada H, Yokoyama A, Hirayama T, Ito R, et al. Metastatic renal cell carcinoma presenting as multiple pleural tumours. Respirology. 2005;10: 128-31.

\title{
The hepatic buttress: A novel surgical technique to treat an intrapericardial diaphragmatic defect after hybrid myocardial ablation
}

\author{
William C. Chapman, Jr, BA, ${ }^{a}$ Michael Sosin, MD, ${ }^{\mathrm{b}}$ J. R. Salameh, MD, FACS, ${ }^{\mathrm{a}, \mathrm{c}}$ and
}

C. Francisco Espinel, MD, FACS, ${ }^{a, c}$ Washington, DC, and Arlington, Va

Multiple medical and interventional therapies are currently available for correcting persistent atrial fibrillation. One such procedure, hybrid ablation, aims to decrease postablative atrial fibrillation relapse by combining transvenous

\footnotetext{
From the ${ }^{\mathrm{a}}$ Georgetown University School of Medicine, Washington, DC; the

${ }^{\mathrm{b}}$ Department of Surgery, Medstar Georgetown University Hospital, Washington, DC; and ${ }^{\mathrm{c}}$ Surgical Specialists, Virginia Hospital Center, Arlington, Va.

Disclosures: Authors have nothing to disclose with regard to commercial support.

Received for publication Feb 1, 2015; revisions received Feb 25, 2015; accepted for publication March 30, 2015; available ahead of print May 7, 2015.

Address for reprints: C. Francisco Espinel, MD, FACS, Virginia Hospital Center, 1625 N George Mason Dr, Suite 334, Arlington, VA 22205 (E-mail: cfespinel@ virginiahospitalcenter.com).

J Thorac Cardiovasc Surg 2015;150:e5-7

$0022-5223 / \$ 36.00$

Copyright (C) 2015 by The American Association for Thoracic Surgery

http://dx.doi.org/10.1016/j.jtcvs.2015.03.065
}

endocardial ablation with surgical scarring of the epicardium. ${ }^{1}$ Recent reports suggest superior outcomes relative to stand-alone transvenous or surgical ablation and an overall complication rate of $4.1 \%$ to $11 \% .^{1,2}$ Reported complications include bleeding, myocardial edema, cardiac tamponade, atrioesophageal fistula, esophageal perforation from thermal injury, and sternotomy. ${ }^{1}$

The following account details a case of herniated small bowel within an intrapericardial diaphragmatic defect after hybrid myocardial ablation through a subxiphoid approach, ultimately resulting in small-bowel obstruction. Treatment involved laparoscopic reduction of herniated bowel, primary repair of the defect, and buttressing the closure with the left triangular ligament of the liver. The purpose of this case is to highlight a previously unreported 


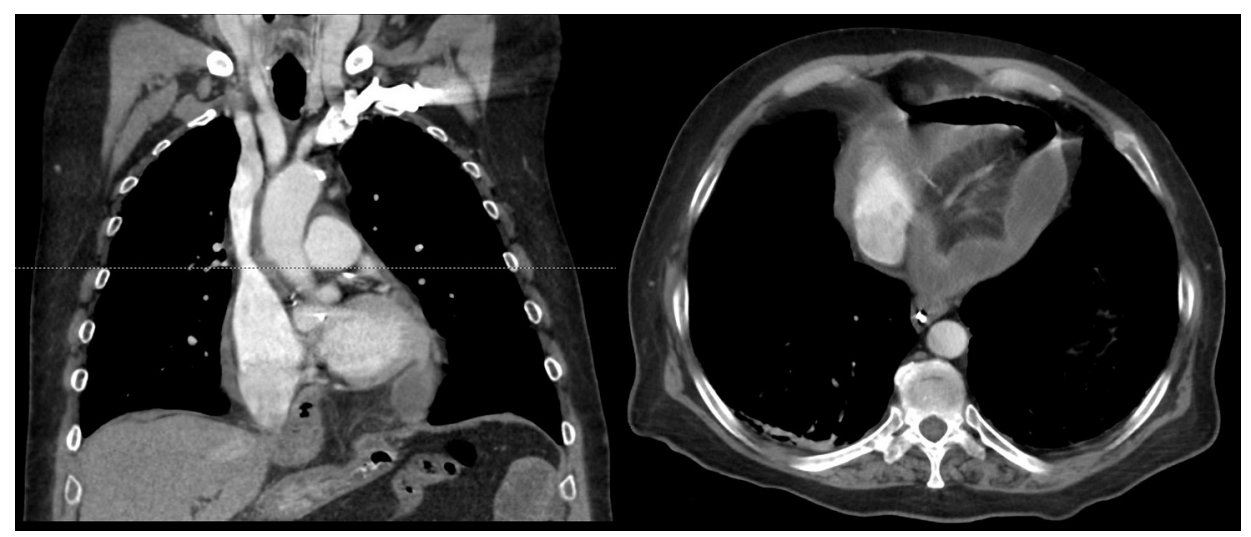

FIGURE 1. Computed tomographic coronal (left) and transverse (right) cuts demonstrating herniation of bowel and fat through the diaphragmatic defect into the pericardial space.

complication after hybrid ablation and to describe a novel surgical technique for diaphragmatic repair.

\section{CLINICAL SUMMARY}

A 69-year-old white man with a history of chronic obstructive pulmonary disease and atrial fibrillation after myocardial hybrid ablation was seen with "cramping" diffuse abdominal pain, anorexia, multiple episodes of emesis, and constipation. Computed tomography of the chest and abdomen confirmed a diagnosis of small-bowel obstruction with herniation of bowel and fat through a diaphragmatic defect into the pericardial space (Figure 1).

Medical record review revealed that 4 months previously the patient had undergone hybrid laparoscopic and transvenous myocardial ablation for treatment of increasingly symptomatic, persistent ( $>10$ years) atrial fibrillation refractory to 3 cardioversions and trials of dofetilide and sotalol. During that procedure, the diaphragm was incised lateral to the central tendon for laparoscopic access to the pericardial sac. The posterior left atrial epicardium was then ablated with radiofrequency energy delivered by an EPi-Sense Guided Coagulation Device (nContact, Inc, Morrisville, NC), and a pericardial drain was brought out through the diaphragmatic defect. The patient was then transferred to the electrophysiology suite for transvenous endocardial ablation. At the conclusion of the combined procedure, the patient was in normal sinus rhythm, with the diaphragmatic defect left unrepaired.

Concern for incarcerated bowel becoming strangulated prompted diagnostic laparoscopy. On entry of the abdomen, a posterior midline defect in the diaphragm was visualized, with incarcerated omentum and 2 loops of small bowel. Gentle traction and limited lysis of adhesions allowed the hernia contents to be reduced from the pericardial space, revealing a $3-\mathrm{cm}$ diaphragmatic defect with thickened and chronically inflamed edges that could be approximated under gentle traction (Figure 2, A). Inspection of the small
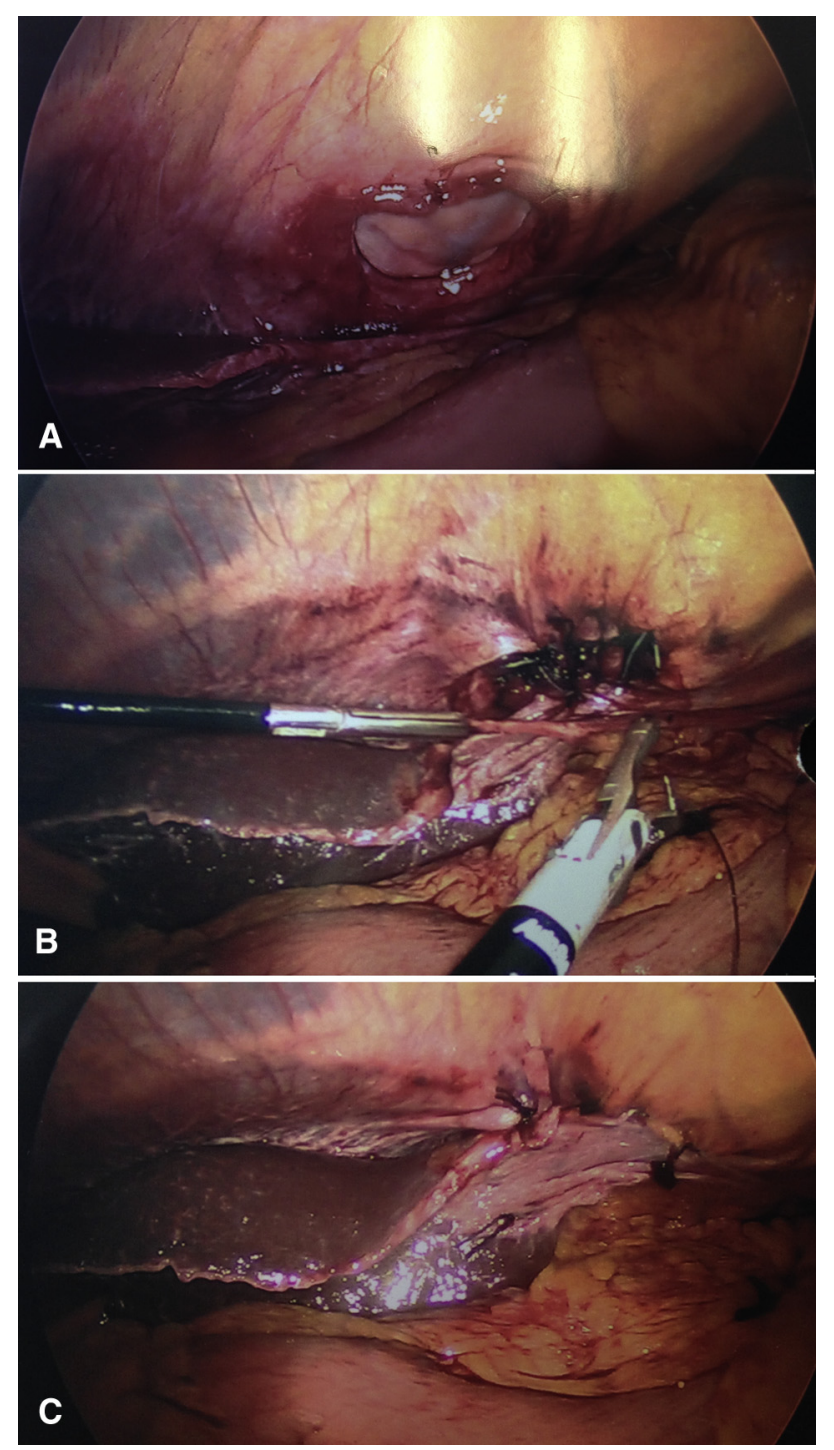

FIGURE 2. A, The defect measured approximately $3 \mathrm{~cm}$. B, Approximation of the defect with interrupted figure-of-eight 1-0 silk sutures. C, Hepatic buttressing of the diaphragmatic repair with the left triangular ligament of the liver. 
bowel revealed dusky tissue without necrosis. The heart was appreciated through the defect.

The diaphragmatic defect was then primarily closed with figure-of-eight 1-0 silk sutures, ultimately leaving the opposing edges approximated (Figure 2, B). To reinforce the closure, the left triangular ligament of the liver was then fixed to the diaphragm overlying the defect by means of interrupted sutures (Figure 2,C). The patient tolerated the procedure well and was discharged home on postoperative day 4. At 1-month follow-up, the patient was without complications and in normal sinus rhythm.

\section{DISCUSSION}

Hybrid transvenous and surgical myocardial ablation is merely the latest iteration of treating atrial fibrillation. ${ }^{2}$ As with many efficacious novel procedures, the safety profile continues to evolve. The largest published data set, a meta-analysis of 9 studies incorporating 335 patients, found a complication rate of $4.1 \% .^{2}$ The most common complications are minor bleeding and cardiac tamponade, but massive hemorrhage, atrioesophageal fistula, and thermal esophageal injury have also been reported. ${ }^{1}$

As demonstrated, unrepaired diaphragmatic defects pose serious risks after hybrid ablation. No consensus exists, however, regarding optimal closure of intrapericardial diaphragmatic defects. Although small defects can often be closed primarily, ${ }^{3}$ larger defects prove more challenging. Synthetic mesh repair, the criterion standard for large diaphragmatic defect repair, should be avoided in cases of increased risk for infection. ${ }^{3}$ Biologic meshes have been used in such cases ${ }^{3}$ but still have a propensity for complications, including infection, seroma, erosion, and potential explantation. ${ }^{4}$ The use of autologous vascularized tissue performs well in conditions conducive to infection. ${ }^{5}$

The hepatic buttress provides multiple benefits as a diaphragm closure technique without the complications of stand-alone primary or mesh-related repair. Initially, the apertures between sutures can accommodate continuous and passive drainage of effusive fluid, ultimately avoiding life-threatening tamponade. The hepatic ligament reinforces the closure without exposing myocardium or intra-abdominal organs to foreign material, thereby avoiding potential mesh infection, erosion, or dysrhythmia. In addition, the buttress ensures durable closure and greatly reduces the risk of recurrent bowel herniation. The hepatic buttress thus serves as a viable option in treating pericardial diaphragmatic defects, especially after hybrid ablation.

\section{References}

1. Zembala M, Filipiak K, Kowalski O, Boidol J, Sokal A, Lenarczyk R, et al Minimally invasive hybrid ablation procedure for the treatment of persistent atrial fibrillation: one year results. Kardiol Pol. 2012;70:819-28.

2. Gelsomino S, Van Breugel HN, Pison L, Parise O, Crijns HJ, Wellens F, et al. Hybrid thoracoscopic and transvenous catheter ablation of atrial fibrillation. Eur J Cardiothorac Surg. 2014;45:401-7.

3. Al-Nouri O, Hartman B, Freedman R, Thomas C, Esposito T. Diaphragmatic rupture: Is management with biological mesh feasible? Int J Surg Case Rep. 2012;3:349-53

4. Patel KM, Bhanot P. Complications of acellular dermal matrices in abdominal wall reconstruction. Plast Reconstr Surg. 2012;130(5 Suppl 2):216S-24S.

5. Kuo YR, Kuo MH, Lutz BS, Huang YC, Liu YT, Wu SC, et al. One-stage reconstruction of large midline abdominal wall defects using a composite free anterolateral thigh flap with vascularized fascia lata. Ann Surg. 2004;239: $352-8$.

\title{
A case of right atrial compression by the elevated liver successfully treated by diaphragmatic plication
}

\author{
Amr A. Arafat, MD, ${ }^{a}$ Mohamed S. Elkahwagy, MD, ${ }^{a}$ Mohammed H. Sherif, MSc, ${ }^{b}$ and \\ Abdel-Hady M. Taha, MD, ${ }^{\text {a Tanta, Egypt }}$
}

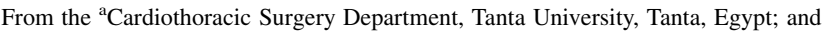
the ${ }^{b}$ Cardiology Department, Tanta University, Tanta, Egypt.

Disclosures: Authors have nothing to disclose with regard to commercial support. Received for publication April 8, 2015; accepted for publication April 18, 2015.

Address for reprints: Amr A. Arafat, MD, Tanta University, Cardiothoracic Surgery Department, Al-Geish St, Tanta, Gharbyia 31529, Egypt (E-mail: amr.arafat@ med.tanta.edu.eg).

J Thorac Cardiovasc Surg 2015;150:e7-10

$0022-5223 / \$ 36.00$

Copyright (C) 2015 by The American Association for Thoracic Surgery

http://dx.doi.org/10.1016/j.jtcvs.2015.04.053
}

Diaphragmatic eventration in old age is a rare anomaly that infrequently requires intervention. The etiology of the eventration could be congenital in origin as a result of failure of development of the muscle of the diaphragm or less frequently secondary to acquired conditions. ${ }^{1,2}$ In most cases diaphragmatic eventration is asymptomatic, with incidental discovery on chest radiography, or it may present with dyspnea, chest infection, gastrointestinal symptoms, ${ }^{2}$ and, rarely, cardiac compression symptoms. ${ }^{3}$ 